\title{
Atención a la diversidad en la Formación Profesional.
}

(Attention to diversity in professional training.)

\author{
Alejandro Luís Álvarez Mercado \\ Profesor de Enz. Secundaria Esp. Organización y Proyectos de Fabricación Mecánica. \\ Ingeniero Industrial (Universidad de Jaén y \\ Fachhochschule Offenburg, Alemania)
}

Fecha recepción: 11-08-2018

Páginas 175-180

Fecha aceptación: 30-09-2018

\section{Resumen.}

El objetivo principal de este trabajo es resaltar la importancia que tiene la atención a la diversidad en la Formación Profesional. Es sabido que la Formación Profesional, no es una enseñanza obligatoria, pero no por ello debemos olvidar a aquellos alumnos y alumnas que precisen de algún tipo de medida de atención a la diversidad, de manera que puedan acceder a una formación adecuada que le permita su futura inserción al mundo laboral.

Asimismo, y teniendo en cuenta que la legislación que regula las enseñanzas de Formación Profesional, no permite la modificación ni supresión de objetivos, contenidos, ni resultados de aprendizaje, en este artículo se van a plantear diferentes medidas que nos permitan dar respuesta a los alumnos y alumnas que presenten necesidades específicas de apoyo educativo.

Palabras-clave: atención a la diversidad; formación profesional

\section{Abstract.}

The main objective of this work is hihglight the importance that has the attention to diversity in professional training. It is well known that profesional training is not a compulsory education, but we should not forget the students that need some kind of measure to attention to diversity, so that they can acces appropriate training, which allows them to enter the labor market.

Also, taking into account that the legislation of the profesional teachings does not allow the modification or suppression of objectives, contents, or learning outcomes, in this article different measures will be proposed a different measures which will allow us to respond to students who have specific needs for educational support.

Keywords: attention to diversity; profesional training 


\section{1.-Introducción.}

La diversidad es una característica intrínseca de los grupos humanos, ya que cada persona tiene un modo especial de pensar, de sentir y de actuar, independientemente de que, desde el punto de vista evolutivo, existan unos patrones cognitivos, afectivos y conductuales con ciertas semejanzas. Dicha variabilidad, ligada a diferencias en las capacidades, necesidades, intereses, ritmo de maduración, condiciones socioculturales, etc., abarca un amplio espectro de situaciones, en cuyos extremos aparecen los sujetos que más se alejan de lo habitual. Frente a una visión que asocia el concepto de diversidad exclusivamente a los colectivos que tienen unas peculiaridades tales que requieren un diagnóstico y una atención por parte de profesionales especializados, consideramos que en los grupos educativos existe una variabilidad natural, a la que se debe ofrecer una atención educativa de calidad.

Por tanto, entendemos la atención a la diversidad como el conjunto de acciones educativas que en un sentido amplio intentan prevenir y dar respuesta a las necesidades, temporales o permanentes, de todo el alumnado del centro y, entre ellos, a los que requieren una actuación específica derivada de factores personales 0 sociales relacionados con situaciones de desventaja sociocultural, de altas capacidades, de compensación lingüística, comunicación y del lenguaje 0 de discapacidad física, psíquica, sensorial o con trastornos graves de la personalidad, de la conducta o del desarrollo, de graves trastornos de la comunicación y del lenguaje de desajuste curricular significativo

\section{2.-Diversidad.}

"Se entiende por diversidad todas aquellas características excepcionales del alumnado, provocadas por diversos factores que requieren una atención especializada para que todo el alumnado alcance un mismo nivel de aprendizaje" (Silva, 2007, p.3). Hoy en día, el concepto de atención a la diversidad en la escuela está muy ligado a las necesidades específicas de apoyo educativo. Siguiendo las publicaciones de Ruiz (2010), sostenemos que: La atención a la diversidad es un concepto amplio que incluye las dificultades de aprendizaje, discapacidades físicas, psíquicas y sensoriales, los grupos de riesgo, las minorías étnicas, etc. El concepto de diversidad nos plantea que todos los alumnos tienen unas necesidades educativas individuales propias y específicas para poder acceder a las experiencias de aprendizaje necesarias para su socialización, establecidas en el currículo escolar.

Según González, Martín, Flores, Jenaro, Poy y Gómez (2013), la diversidad es un potencial enriquecedor para todos los individuos involucrados. En este mismo sentido, se expresa que el contexto educativo como sistema ofrece a los estudiantes: contenidos académicos adaptados a las necesidades educativas, interacciones colaborativas entre los miembros; para estimular, fomentar, ampliar las oportunidades de aprendizaje y erradicar barreras para su inclusión (Jiménez, Lalueza y Fardella, 2017) 


\section{3.-Integración.}

Birch (1974), citado por Bautista (1993), la define como un proceso que pretende unificar las educaciones ordinaria y especial con el objetivo de ofrecer un conjunto de servicios a todos los niños, en base a sus necesidades de aprendizaje" (Bautista, 1993, p.31), Kaufman (1975), citado por Sanz del Río (1986) la define como: La integración temporal, instructiva y social de un grupo de seleccionado de niños excepcionales, con sus compañeros normales, basada en una planificación educativa y un proceso 'programador evolutivo e individualmente determinado. Esta integración requería una clasificación de responsabilidades entre el personal educativo regular y especial y el personal administrativo, instructor y auxiliar. (p.27).

Más adelante, en el año 1996, Tony Booth la define como un proceso consistente en responder a la diversidad de necesidades de todos los alumnos y satisfacerlas mediante una mayor participación en el aprendizaje, las culturas y las comunidades, así como en reducir la exclusión dentro de la educación y a partir de ella.

La NARC (National association of Retarded Citizens, USA) la define como: "la integración es una filosofía o principio de ofrecimiento de servicios educativos que se pone en práctica mediante la provisión de una variedad de alternativas instructivas y de clases, que son apropiadas al plan educativo, para cada alumno, permitiendo la máxima integración instructiva, temporal y social entre alumnos deficientes y no deficientes durante la jornada escolar normal".

\section{4.-Inclusión.}

La UNESCO (2008) la define como: Un proceso de abordaje y respuesta a la diversidad de las necesidades de todos los alumnos a través de la creciente participación en el aprendizaje, las culturas y las comunidades, y de la reducción de la exclusión dentro y desde la educación. (p.11) Según esa misma Conferencia Internacional de Educación de 2008, el concepto de inclusión ha evolucionado hacia la idea que todos los niños, las niñas y los jóvenes deberían tener condiciones y oportunidades equivalentes de aprendizaje en diferentes tipos de escuelas independientemente de sus antecedentes sociales y culturales, así como de sus diferencias en las habilidades y capacidades.

La recomendación internacional insiste en la necesidad de plantear la educación inclusiva como algo inacabado, que empieza en los primeros años de vida y se mantienen hasta que se acaba (Casanova, 2011, p. 95). De este modo, la función de las escuelas es ofrecer las herramientas acordes a sus características particulares, de manera que todos y cada uno de los estudiantes de la comunidad educativa se sientan acogidos, seguros y convencidos de que lograrán sus metas. Por ello, la inclusión educativa se considera un proceso de cambio que permite abordar las necesidades de los estudiantes con alguna diversidad, pues tiene su base en los derechos humanos pro-moviendo la equidad y justicia social (Fasting, 2013; Mäkinen, 2013). Se construye así la capacidad que debe tener la escuela inclusiva de aceptar 
a todos los alumnos que deseen participar en ella y, de este modo, reducir la exclusión de los mismos (R. Á., Contreras, C. R. 2018).

\section{5.-Atención a la diversidad y formación profesional.}

Partimos de la premisa de que la Atención a la Diversidad es un principio que debe regir toda la enseñanza para proporcionar a todo el alumnado una educación adecuada a sus características y necesidades. El sistema educativo debe procurar medidas flexibles que se adecuen a las diferencias individuales y ritmos de maduración de cada uno de los alumnos y alumnas.

La atención a la diversidad requiere un enfoque inclusivo, el cual, busca promover en el profesorado una formación teórico-práctica, configurando actitudes favorables hacia la diversidad e inclusión; y con el conocimiento del desarrollo de las personas con necesidades educativas asociadas a la discapacidad (García, Massani y Bermúdez, 2016).Medina, De la Herrán y Sánchez (2011) plantean que, bajo este contexto educativo, los profesores deben estar preparados para asumir, también, su rol de líderes sociales en la atención a la diversidad. Por lo tanto, es importante que, desde el nivel de educación básica hasta la educación superior, el profesorado cuente con una formación adecuada que dé respuesta a las necesidades de los estudiantes; así como contar con constantes capacitaciones que permitan dentro del aula resolver conflictos, propiciar una comunicación y motivación en los alumnos (Serrano y Pontes, 2017).

La diversidad del alumnado constituye una realidad en los centros educativos y ha de ser asumida por todo el profesorado con criterios de normalización, atención personalizada e inclusión. El Plan de Atención a la Diversidad que los centros elaboran recoge las medidas organizativas ordinarias y extraordinarias para dar respuesta a las necesidades educativas de todo el alumnado.

Asimismo, debemos de tener en cuenta el nivel educativo en el que nos encontramos, en este caso Formación Profesional, la cual, Según el Real Decreto $1147 / 2011$, de 29 de julio, por el que se establece la ordenación general de la formación profesional del sistema educativo, se define como el conjunto de acciones formativas que tienen por objeto la cualificación de las personas para el desempeño de las diversas profesiones, para su empleabilidad y para la participación activa en la vida social, cultural y económica.

Como cualquier etapa educativa, la formación profesional consta de una evaluación, a través de la cual se valora en qué medida y si son superados o no los contenidos básicos establecidos para dicha etapa. Para ello hay que tener en cuenta la orden 29 de septiembre de 2010, por la que se regula la evaluación, certificación, acreditación y titulación académica del alumnado que cursa enseñanzas de formación profesional inicial que forma parte del sistema educativo en la Comunidad Autónoma de Andalucía. Concretamente el artículo 2. e) en el que se hace referencia al alumnado con necesidades específicas de apoyo educativo, establece: "La adecuación de las actividades formativas, así como de los criterios y los procedimientos de evaluación 
cuando el ciclo formativo vaya a ser cursado por alumnado con algún tipo de discapacidad, garantizándose el acceso a las pruebas de evaluación. Esta adaptación en ningún caso supondrá la supresión de resultados de aprendizaje y objetivos generales del ciclo que afecten a la adquisición de la competencia general del título."

De este modo teniendo en cuenta lo establecido por la ley se ajustará la ayuda pedagógica a las diferentes necesidades del alumnado y se facilitarán recursos 0 estrategias variadas que permitan dar respuesta a la diversidad del aula.

\section{6.-Actuaciones como respuesta a la atención a la diversidad en FP.}

Entre las actuaciones o vías que se plantean para el tratamiento de la diversidad podemos encontrar las siguientes:

- Se emplearán metodologías didácticas diferentes, que se adecuen a los distintos grados de capacidades previas, a los diferentes niveles de autonomía y responsabilidad de los alumnos y a las dificultades o superlogros detectados en procesos de aprendizaje anteriores.

- Se adaptarán las actividades a las motivaciones y necesidades del alumnado, planteando aquéllas a un nivel situado entre lo que ya saben hacer autónomamente y lo que son capaces de hacer con la ayuda y guía del profesor 0 de sus compañeros. Se establecerá de menor a mayor la dificultad de las tareas, de tal forma que todos los alumnos puedan encontrar espacios de respuesta adecuados a sus capacidades.

- Las actividades/prácticas grupales propuestas se desarrollarán en grupos heterogéneos, prestando atención al reparto de tareas y a una asignación de funciones flexible.

- Se respetarán los ritmos de aprendizaje de los alumnos, en función de sus intereses, motivaciones y capacidades.

- En las actividades de recuperación programadas, para los alumnos con más dificultades de aprendizaje se definirán de manera clara los conceptos que les cuesta trabajo comprender. Se plantearán estas actividades teniendo presente los niveles mínimos que habrán de alcanzar los alumnos.

- Se plantearán actividades de ampliación para los alumnos que pueden ampliar el proceso de aprendizaje.

- Se mejorará el hábito de estudio y la responsabilidad.

- Se fomentarán las relaciones sociales y la integración de los alumnos del grupo.

\section{7.-Conclusión.}

Partiendo de todo lo expuesto anteriormente, debemos recalcar la importancia que tiene una buena respuesta educativa a las necesidades que pueda presentar el alumnado. Para dar esta repuesta es necesario tener en cuenta los términos de inclusión, diversidad e integración, ya que serán la base para poder atender a toda la diversidad de alumnos y alumnas presentes en el aula. 
Debido a que nos encontramos en la etapa de enseñanzas profesionales es fundamental ofrecer una igualdad de oportunidades a todo el alumnado, ya que facilitara su futura inserción a nivel social y por supuesto al mundo laboral.

El objetivo principal de las enseñanzas profesionales es la incorporación al mundo laboral de los alumnos y alumnas, es aquí donde debemos destacar la importancia de la actuación docente, para que este objetivo no se vea truncado.

Es sabido que para obtener el título de formación profesional los alumnos y alumnas deben superar una serie de módulos, los cuales constan de unos objetivos, contenidos, criterios de evaluación, etc. Según la ley los elementos básicos del currículo no pueden sufrir modificación alguna, pero sí, elementos como puede ser la metodología, entre otros. Por ello en este artículo se ha planteado una serie de actuaciones a emplear con el alumnado que así lo requiera.

\section{8.-Bibliografía.}

Agencia europea para el Desarrollo de la Educación Especial (2003). Necesidades Educativas Especiales en Europa. Publicación Monográfica volumen 1.

Ainscow, M., Booth, T., et. al. (2002). Índice de inclusión; desarrollando el aprendizaje y la participación de las escuelas. Santiago: CISIE.

Araque, H. y Puente, B. (2010). Atención a la diversidad y desarrollo de procesos educativos inclusivos. Revista de Ciencias Sociales nº4.

Armstrong, F. (1999). Inclusion, curriculum and the struglle for space in school. International Journal of Inclusive Education.

Arnáiz Sánchez, P. (2003). Educación inclusiva: una escuela para todos. Málaga: Ediciones Aljibe.

Bautista Jiménez, R. (1993). Necesidades educativas especiales. Málaga: Ediciones Aljibe.

Booth, T. (1996). A Perspective on Inclusion from England. Cambridge Journal of Education, 26(1), 87-99.

Echeita, G. (1988). Profesores y otros profesionales para la atención de los alumnos con N.E.E. Serie Documentos, № 7. Madrid: Centro Nacional de Recursos de E.E.

Fernández González, G.M. (1993). Teoría y análisis práctico de la integración. Madrid: Escuela Española. 